\title{
1 Foyer und Pause, Interaktion und Kontext: Zur Einleitung in den Band
}

\author{
unter Mitarbeit von Jan Gerwinski, Christine Hrncal \& \\ Eva Schlinkmann
}

\subsection{Problemhintergrund und Ausgangsfragen}

Foyer- oder Pausengespräch? - Für den Typus der Gespräche, die im Mittelpunkt der folgenden Untersuchungen stehen, erscheinen beide Bezeichnungen gleichermaßen treffend. Die Art der Interaktion, die sich bei derartigen Gelegenheiten entfaltet, ist nämlich ebenso mit den besonderen örtlichen wie mit den spezifischen zeitlichen Umständen des Ereignisses eng verwoben. ${ }^{1}$ Diese zeiträumlichen und dinglichen Charakteristika der Situation werden in einer praxistheoretischen Perspektive, wie sie dem vorliegenden Band zugrunde liegt (vgl. Kapitel 3, i.d.B.), nicht als äußerliche, determinierende Bedingungen betrachtet (Deppermann, Feilke \& Linke 2016a, 5), sondern als Bestandteile der kommunikativen (und über Kommunikation hinausreichenden) und zugleich soziokognitiven Praktiken, deren sinnhafte, individuell zugerechnete Einheiten ,Handlungen“ und ihre kulturellen Verstehenshintergründe/Kontexte sind: Praktiken

(1) help institute which mental states and actions humans are and can be in and (2) are the contexts in which humans acquire the wherewithal to be in these states and to perform the actions that compose practices (Schatzki 1996, $12 \mathrm{f}$.). ${ }^{2}$

1 Wie im Prinzip jede ,soziale Veranstaltung،, vgl. Günthner \& Knoblauch 1994, 711; Linz, Hrncal \& Schlinkmann 2016, 528. Zum Zusammenhang von (multimodaler) Interaktion und Raum vgl. - stellvertretend für einen umfangreiches, seit einigen Jahren stark wachsendes Korpus an Fachliteratur - exemplarisch Hausendorf, Schmitt \& Kesselheim 2016.

2 Auf der „flachen“ ontologischen Basis einer derartigen Praxistheorie (Schatzki 2016), mit der die notorisch unbefriedigende sozialtheoretische Polarität von „Makroebene“ (langfristig stabile soziale Strukturen, Technologien, Systeme von Werten, Zeichen etc.) und „Mikroebene“ (individuelles Handeln) zu überwinden ist, können ,Handlungen', ihre situationale, raumzeitliche und dingliche Einbettung und ihre situativ relevanten ,Kontexte - ausgehend von dem in Praktiken eingebetteten Sprach- und Zeichengebrauch im Rahmen einer interaktionalen „Infrastruktur“ (Schegloff 2006/2012) - interpretativ rekonstruiert werden. Praktische Beschränkungen ergeben sich durch die notwendige, je nach Methodenkombination und Technikeinsatz unterschiedlich weit reichende Selektivität der Datenerhebung, wobei das unerreichbare 
Der Ort, das Theaterfoyer, ist ein öffentlicher, im Unterschied etwa zum privaten Wohnzimmer beim gemeinsamen Fernsehen in der Gruppe (vgl. Holly, Püschel \& Bergmann 2001). Mit dem Konzept der Öffentlichkeit bzw. des Publikums verbindet sich nach einer - auch heute noch publizistisch vertretenen - Idealvorstellung des Theaters nicht nur die Vorstellung einer (lokal versammelten) Menschenmenge, die von den Veranstaltern einer öffentlichen Darbietung oder Ausstellung als „Zielgruppe“ (ökonomisch) adressiert wird (Kernbauer 2012, 63; Habscheid, Hrncal, Knipp \& Linz 2016a, 464). Vielmehr gilt das Theaterfoyer, aller kulturkritischen Skepsis zum Trotz, vielfach noch immer als „,Podium“ einer bürgerlichen Öffentlichkeit, [...] eines ,räsonierenden Publikums““, das sich über ästhetische und gesellschaftspolitische Probleme verständigt (Habermas 1962/1990, 69, 96; Linz, Hrncal \& Schlinkmann 2016, 524, mit publizistischen Belegen für die Gegenwart).

Zum Gegenstand und/oder Anlass des Räsonnements, dies liegt buchstäblich nahe, kann im Foyergespräch nicht zuletzt das werden, was sich gerade zuvor auf einem kleineren Podium im größeren ereignete: die Aufführung auf der Bühne vor anwesendem Publikum im Saal. Goffman spricht vom ,Spiel‘ als dem inneren Rahmen des Theaters, der in den äußeren Rahmen des ,Spektakulums', des Theaters als eines sozialen Ereignisses, eingebettet ist (Goffman 1977, 149 ff. und 287ff; Linz, Hrncal \& Schlinkmann 2016, 525 f.). In diesem Sinn lassen sich, nach dem Modell eines wechselseitigen Konstitutionsverhältnisses, Foyergespräche auch als eine Art alltägliches Theater verstehen (vgl. zu den Quellen einer derartigen Metaphorik den Überblick von Buss 2009), das sich räumlich und zeitlich um ein künstlerisches Theater herum entfaltet, ${ }^{3}$ diesem einen institutionellen Rahmen schafft, umgekehrt hiervon angeregt wird und sich teilweise thematisch hierauf bezieht.

Der Ausdruck Theater im Titel des Projekts kann also sowohl wörtlich als auch metaphorisch verstanden werden, mit Referenz zum einen auf mögliche Themengebiete derartiger Gespräche (Sprechen über das Theater; aus Anlass des Theaters), zum anderen auf den ,theatralen' Charakter derartiger Gespräche selbst (alltägliches Sprechen in der Öffentlichkeit als Theater). Eine Brücke zwischen beiden Ebenen schlägt - mit Blick auf das gesellschaftliche Funktionsprinzip des Theaters ${ }^{4}$ - Hans Georg Soeffner:

Ideal einer semiotischen Vollständigkeit nicht zum Fetisch gemacht werden sollte.

3 Zur räumlichen und zeitlichen Abgrenzung (durch Vorhang, Beleuchtung etc.) vgl. Linz, Hrncal \& Schlinkmann 2016, 525, unter Bezug auf Goffman 1977 sowie Kapitel 3, i.d.B.

4 Die in gewisser Hinsicht der wissenschaftlichen Reflexion von Interaktion vergleichbar ist, allerdings von anderen Mitteln der perspektivischen Brechung Gebrauch macht. Vgl. hierzu 
Theater und Schauspiel machen darauf aufmerksam, daß wir nicht nur in ihnen, sondern auch in unserem Alltag, virtuelle Zuschauer unserer selbst und der Welt' sind und daß wir als solche immer auch die ,Welt in Szene setzen' (Soeffner 2001, 168).

Auf dieser Basis stehen im Idealfall die Kommunikation auf der Bühne, die Kommunikation durch das Bühnengeschehen und die Kommunikation im Anschluss daran - noch weit über das Foyergespräch hinaus - in einem engen Zusammenhang: ${ }^{5}$ „Was ich von einem Abend erwarte, ist relativ simpel: Ich möchte gut unterhalten und gepackt werden, so dass ich mich noch lange über das Stück unterhalten kann." So lautete die Antwort des ehemaligen Bürgermeisters von Berlin in einem Interview der „Frankfurter Allgemeinen Zeitung“ auf die Frage des Journalisten: „Noch mal zu Ihnen als Zuschauer: Wenn Sie ins Theater gehen, was erwarten Sie von einem Abend dort?“ (Strauß 2017).

Bereits im Blick auf das Bühnengeschehen können eine Ebene der Darstellung von der Ebene des Dargestellten und damit auch hier ein ,äußerer'von einem ,inneren` Rahmen unterschieden werden (Goffman 1977, 149; Linz, Hrncal \& Schlinkmann 2016, 526). Für das Dargestellte kann weiter die ,Welt des Stücks' von Bezugnahmen auf die ,Welt außerhalb des Theaters' unterschieden werden; diese und weitere Ebenen können - mit fließenden Übergängen im Rahmen einer dynamischen Praxis - in der anschließenden Interaktion der Zuschauer aufgegriffen bzw. hergestellt werden (Hrncal \& Gerwinski 2015; Kapitel 6, i.d.B.).

Das Theaterfoyer und ein Außenbereich sind bereits seitens der Institution als Ort für semi-öffentliche Interaktionen im Publikum hergerichtet, etwa durch eine Möblierung, die es kulturell ermöglicht und nahelegt, sich in kleinen Gruppen, die sich in der Regel bereits vor dem und für den - oftmals gemeinsamen - Theaterbesuch konstituiert haben, im und im Umfeld des Theatergebäudes zum Gespräch zu versammeln (vgl. Kapitel 6, i.d.B.). In solchen Situationen befinden sich andere Besucher - mit unterschiedlichen, auch rahmengebunden wechselnden Beteiligtenstatus (vgl. Goffman 1981a) - zumeist zumindest in prinzipieller Hörweite („,natural“ surveillance“, vgl. Bergmann 1990, 219).

Zugleich verweist der Ort auch auf den Pausencharakter der hier stattfindenden Aktivitäten, die typischerweise Kommunikation umfassen, aber auch weit darüber hinaus - in den Bereich primär physischer Praktiken hinein -

auch Baecker 2013, 17.

5 Vgl. zu einer ähnlichen Konzeption im Blick auf die Bildende Kunst, bei der Kommunikation durch Kunst und mit Kunst und die Kommunikation über Kunst (durch Museen, Kritiker, das Publikum etc.) ineinander greifen, Hausendorf \& Müller 2016a. 
reichen (s.o.): ${ }^{6}$ Gastronomische Verpflegung, die Möglichkeit, sich miteinander essend, trinkend und sprechend um einen Bistro-Tisch herum zu positionieren, Gelegenheiten zum gemeinsamen Gehen im Innen- und Außenbereich legen nahe, dass die Pause nicht nur einen Rahmen für andere Aktivitäten, etwa das Theatergespräch, bietet, sondern nicht zuletzt auch als Pause, also für Praktiken der Erholung, Entspannung, Erfrischung, Stärkung etc. genutzt werden kann, wobei Übergänge zwischen unfokussierter und fokussierter Interaktion (vgl. Goffman 1963, 24) zu gestalten sind (vgl., am Beispiel eines Empfangs, Ruoss 2014; zu einer ähnlichen Situierung in der Peer Group-Kommunikation Jugendlicher vgl. Deppermann \& Schmidt 2001, 29). Dass der eigentliche Theaterbesuch auch anstrengend sein kann, gehört zu den gängigen Topoi im Pausengespräch.

Mit dem ungezwungeneren Charakter von Pauseninteraktion korrespondieren auf der Ebene der sprachlichen Formate Gattungen des Small Talks und der Konversation, einer - dem Stereotyp nach - „von Kaviarbrötchen und Rosésekt eingerahmten Gesprächsform“ (Linke 1988, 140 f.), z.T. auch der so genannten „homilëischen Diskurse“ (vgl. Kapitel 5, i.d.B.). Unter dem Begriff „,homilëischer Diskurs“ werden in der „Funktionalen Pragmatik“ Interaktionstypen, etwa am Arbeitsplatz, gefasst, die sich außerhalb institutioneller Zwecke, formeller und professioneller Rollen vollziehen und die dennoch für die Reproduktion der Institution einen wichtigen, weil komplementären Beitrag leisten (vgl. etwa Rehbein 2012, 85): In homilëischen Diskursen, z.B. beim Lästern über den Chef oder Lehrer, wird spielerisch-kreativ ,verhandelt [...], was ,in der Welt [der Institution] nicht aufgeht““ (vgl. etwa Rehbein 2012, 89). Als typische Situationen, die den reproduktiven Charakter dieser Art von Kommunikation erkennen lassen, werden Pausengespräche am Arbeitsplatz oder in der Schule (vgl. dazu auch Könning 2015) genannt, die teilweise an eigens von der Institution dafür bereitgestellten Orten stattfinden (Pausenhof, Lehrerzimmer usw.), andererseits in der offiziellen Perspektive der Institution aber auch leicht übersehen werden. Derartige Formate, z.B. theatrale, parodistische Reinszenierungen von Teilen der Vorstellung, sind auch für Pausengespräche im Theater charakteristisch (vgl. Kapitel 8, i.d.B.).

Hierbei kommen auch - in Abhängigkeit vom Wissen der Beteiligten - die spezifischen zeitlichen Umstände des sozialen Ereignisses zum Tragen: Das zeitliche Budget ist durch die Institution bekanntlich begrenzt, aber zumindest für das Publikum typischerweise nicht exakt vorhersehbar, vielleicht auch nicht

6 Vgl. zeichentheoretisch-grundlegend, zur ,symphysischen“ und, empraktischen“ Einbettung von Sprachgebrauch, Bühler 1934/1982, $154 \mathrm{ff}$. 
von vornherein präzise geplant. Dieser Umstand kann bei der individuellen und interaktionalen Gestaltung der Pausen-Aktivitäten prophylaktisch ins Kalkül gezogen werden (vgl. Kapitel 7, i.d.B.), und/oder man stellt sicher, dass die Abfolge von Gong-Zeichen, die das Zu-Ende-Gehen der Pause markieren, situativ wahrgenommen und dann im Blick auf eine Beendigung der Interaktion mehr oder weniger spontan verarbeitet werden kann.

Die räumlichen und zeitlichen Bedingungen der Situation bleiben den Gesprächen nicht äußerlich: Nahe gelegt (und typischerweise aufgegriffen) werden, wie wir gesehen haben, zum einen Praktiken, die, ergänzend zum interaktiven Geschehen im Theatersaal, unter Bedingungen zeitlicher Begrenztheit ein Theater-Publikum konstituieren - und damit das Theater selbst, das ohne ein Publikum nicht möglich ist (Goffman 1977, 144; Linz, Hrncal \& Schlinkmann 2016, 525). Zum anderen bieten Foyer und Pause Gelegenheit, sich zu erholen und zu entspannen - bis hin zum geselligen Ausgehvergnügen, bei dem die Pause und das Beisammen-Sein in einem gastronomischen Rahmen im Anschluss an die Vorstellung u.U. den eigentlichen Grund der Aktivität darstellen und wichtiger sein können als die Theatervorstellung selbst (Goffman 1977, 296; Linz, Hrncal \& Schlinkmann 2016, 526). Derartige durch Konversation geprägte soziale Situationen - wie der gemeinsame Theaterbesuch - entsprechen „Lebensformen und Freizeitbeschäftigungen“, die traditionell mit ,bürgerlicher Geselligkeit assoziiert sind (Linke 1988, 134).

Diese eher soziologisch als ästhetisch relevanten Charakteristika stellen vielleicht einen Grund dafür dar, dass über das Kunstpublikum und seine Alltagspraktiken (Habscheid, Hrncal, Knipp \& Linz 2016) - aller theoretischen Relevanz der „Entdeckung des Zuschauers“ (Fischer-Lichte 1997) in Rezeptionsästhetik und Theaterwissenschaft zum Trotz - bisher erstaunlich wenig bekannt ist (Kammerer 2012a; Linz, Hrncal \& Schlinkmann 2016, 525). Die vorliegenden Studien konzentrieren sich auf den Bereich der bildenden Kunst (vgl. Hausendorf \& Müller 2016a), für den Bereich der prozessual-performativen Kunst liegen hingegen noch keine systematisch ausgearbeiteten Untersuchungen zur Publikumskommunikation vor. Insbesondere fehlt es an Arbeiten, die sich der konstitutiven Rolle des Publikums für die Kunst aus empirischer Perspektive widmen (vgl. Hausendorf \& Müller 2016a, 29, 42f.). Vor diesem Hintergrund fragte das Forschungsprojekt „Theater im Gespräch. Sprachliche Kunstaneignungspraktiken in der Theaterpause", das von der Deutschen Forschungsgemeinschaft von April 2014 bis März 2016 gefördert wurde ${ }^{7}$ und dessen Ergebnisse - ergänzt um weitere Befunde - mit dem vorliegenden Band vorgestellt werden, nach den

7 DFG-Geschäftszeichen: HA 2850/4-1. 
sprachlich-kommunikativen Alltagspraktiken des Publikums im Foyer-/Pausengespräch.

Ziel des Projekts war eine empirische, datengeleitete Rekonstruktion des für derartige Gespräche charakteristischen sprachlich-kommunikativen Repertoires. Anknüpfend an eine Pilotstudie mit Unterstützung des Berliner Theaters „Hebbel am Ufer“ und des Siegener „Apollo-Theaters“ wurde mittels Audiodokumentation - eine Videoaufzeichnung wäre wünschenswert gewesen, schied jedoch wegen des zu invasiven Charakters aus - ein Datenkorpus zu Pausengesprächen von Besuchern künstlerischer Theaterproduktionen im „ApolloTheater Siegen“ und im „Schauspiel Köln“ erhoben (vgl. Kapitel 2, i.d.B.). Anhand dieses Korpus sollten Praktiken der sprachlichen Anschlusskommunikation insbesondere mit Blick auf die folgenden konstitutiven Aspekte analysiert werden:

- sprachlich-interaktionale Verfahren zum Vollzug der spezifischen sozialen Situation des Pausengesprächs als geselliges Ereignis,

- rekonstruktive und transkriptive Verfahren der sprachlichen Bezugnahme auf die ästhetischen Erfahrungen während der Aufführung und

- sprachlich-interaktionale Verfahren der durch das Theater angeregten Reflexion über die eigene Lebenswelt und die gesellschaftliche Wirklichkeit.

Die auf diese Weise zu rekonstruierende, in Routinen der Alltagskommunikation eingebettete Perspektive des Publikums auf das Theater wurde zu anderen Formen und Bereichen der Kunstkommunikation in Beziehung gesetzt. Schließlich sollte das Projekt mit der Erforschung von Sprache in Alltagspraktiken einen Beitrag zu einer praxeologisch fundierten Sprachspiel-/Kulturkonzeption leisten (vgl. Habscheid 2016; Kapitel 3 und 6, i.d.B.).

Es ging dabei von der Annahme aus, dass derartige Gespräche als spezifische soziale Praxis an der Schnittstelle von öffentlicher Kunstinstitution und Geselligkeit sprachliche Aneignungspraktiken von Kunst mit Konversation und „Small Talk“ - metaphorisch: das ,Foyer' mit der ,Pause“ - verbinden. - Wie aber sieht diese Verbindung genau aus? Sind z.B. Kunstgespräch und Small Talk bzw. homilëische Diskurse funktional komplementär, bauen Kunstgespräche in bildungssprachlicher Konversation auf Small Talk gesprächsorganisatorisch und beziehungsbezogen auf, oder existieren beide Typen von Interaktion, ggf. mit fließenden Übergängen, funktional unabhängig nebeneinander? Um es vorweg zu nehmen: Jede dieser drei Annahmen kann empirisch zutreffen.

Nach einer weithin vorherrschenden Annahme, die sich z.T. auch heute noch in den gemeinsprachlichen Bedeutungen von Small Talk und Konversation kristallisiert, erfüllen derartige Gespräche in erster Linie Zwecke der Bezie- 
hungspflege bzw. elitären Abgrenzung, bei gleichzeitiger inhaltlicher Belanglosigkeit (vgl. Kapitel 5.1, i.d.B.). Hiermit korrespondieren theoretische Positionen, die den Sinn derartiger ,bürgerlicher' Konversation heute vor allem auf der sozialstilistischen Ebene verorten und den vorherrschenden Effekt der statusbezogenen Distinktion zu den inhaltlichen „Trivialitäten über Kunst, Literatur oder Film“ in Kontrast setzen (Bourdieu 1979/2013, 284). Auf der anderen Seite ist der exklusive Charakter bildungssprachlicher Konversation aber nur die eine Seite der Medaille. Ebenso deutlich zeigt sich bei näherem Hinsehen, dass für diejenigen, die das Sprachspiel der Gebildeten (vgl. Steinig 2016; populärwissenschaftlich: Schwanitz 1999, 394 ff.) mehr oder weniger beherrschen, bildungssprachliche Konversation, etwa in der Theaterpause, vielfach einen hoch kooperativen Charakter aufweist: Sie hält den internen Wettbewerb um Statuspositionen in der Gruppe und die Demonstration von „Besser- und Anderswissen“ (Holly 2001, 433) dynamisch im Zaum und stellt eine insgesamt ,harmonische' Atmosphäre (vgl. Linke 1988, 140), wechselseitigen Respekt und Anerkennung, das höfliche Vermeiden von Gesichtsbedrohungen und unterhaltsame Geselligkeit in den Vordergrund. Derartige Praktiken werden auf der Ebene der Interaktionsorganisation ermöglicht bzw. begünstigt durch diverse Merkmale von Small Talk und Konversation, die auf deren vergleichsweise ungezwungenen Charakter verweisen.

Bereits in historischer Perspektive, im Blick auf die spannungsreichen Entstehungsbedingungen eines sich aus heterogenen sozialen Gruppen nach und nach herausbildenden ,Bürgertums', werden derartige beziehungsbezogene und gesprächsorganisatorische Merkmale mit der Möglichkeit der Genese und Weitergabe von Bildung in Verbindung gebracht (Bosse 2015, 90; vgl. auch Linke 1988, 140). Praktiken der Konversation gehen also nicht in sozialer Distinktion auf. Vielmehr ermöglicht es der kooperative Charakter von Konversation im Blick auf das institutionelle Aufgabenfeld der - sozial potenziell heiklen (vgl. Müller \& Kluwe 2012a) - Publikums- und Kunstkommunikation, Divergenzen hinsichtlich des Wissens und der Perspektive zu überbrücken und auf dieser Basis voneinander zu lernen. ${ }^{8}$

Für derartige konversationelle Praktiken wird in kulturpessimistischer Perspektive für die Gegenwart ein Verfall behauptet, der u.a. mit dem Wandel kommunikativer Ideale in Verbindung gebracht wird. So betrachtet Stephen

8 Eine spezifische Variante derartiger Kooperationspraktiken kommt in Situationen zum Tragen, in denen Jugendliche im privaten, nicht-schulischen Kontext durch Erwachsene mit den Anforderungen bildungssprachlicher Konversation vertraut gemacht werden (vgl. Kapitel 5.5). 
Miller (2006) in seiner Abhandlung über „Conversation. A History of a Declining Art“ die sprachlichen Praktiken der Gegenwart im Licht philosophischliterarischer Diskurse des 18. Jahrhunderts, als vor allem britische Autoren im Rückgriff auf antike Traditionen Konversation in den Mittelpunkt ihrer sozialen Anthropologie stellten (vgl. zum Folgenden auch Habscheid i.Dr.):

Though the eighteenth-century writers on conversation said that good conversationalists were hard to find, they thought conversation was not only possible, it was also beneficial. Conversation, they said, promotes psychological health and intellectual development. And conversation is one of the great pleasures of life. Several eighteenth-century writers also argued that there is a correlation between political stability and the extent of what Hume calls the 'conversible world.' Like Hume, Addison and Johnson thought that if Britain's educated classes neglected the art of conversation, Britain could become embroiled in violent civil discord. (Miller 2006: xi)

Miller (2006) sieht in Konversation eine auch heute noch erstrebenswerte kulturelle Errungenschaft, die er in einen Gegensatz stellt nicht nur zu Langeweile und Humorlosigkeit, sondern auch zu Parolen, Diskursverweigerung und diktatorischer Unterdrückung $(3,301)$. Sie umfasst - dem Ideal des 18. Jahrhunderts nach - leichte Formen wie den Small Talk (xiv), etwa unterhaltsame Gespräche über Luxus, Mode und gehobene Freizeitbeschäftigungen auf dem Land (16), aber nicht zuletzt auch sprachlich vermittelte Bildungserfahrungen.

Diese hohe Kunst der Konversation sei in der Gegenwart kaum noch zu retten: Während in der Konversation Dissens im Interesse der Sache und des Vergnügens durchaus erwünscht sei, zugleich aber durch Humor, Höflichkeit sowie geistige Unabhängigkeit und Beweglichkeit dynamisch ausbalanciert werde, schwanke - so Miller (2006, 296 f.) - die Kommunikationskultur der Gegenwart zwischen wütendem, erbittertem, vulgärem Streit einerseits (nach dem Motto „Express yourself“), vollkommener intellektueller Indifferenz gegenüber unterschiedlichen Positionen andererseits (nach dem Prinzip „Don’t be judgemental“). Schließlich drängten Praktiken der Mediennutzung die Konversation als Mittel der Welterschließung, Persönlichkeitsentwicklung und Beziehungskonstitution zunehmend zurück (vgl. Miller 2006, 20, 297), während kulturelle Gegenbewegungen jedenfalls in den USA vergleichsweise marginal seien.

Es war anhand unseres Datenmaterials empirisch zu prüfen, ob sich in der Gegenwart Sozialformen finden, die sich an dem Ideal des 18. Jahrhunderts mehr oder weniger messen lassen (was wir, cum grano salis, bejahen würden). 


\subsection{Material und Methoden: Die Durchführung des Projekts}

Dem Projekt ${ }^{9}$ ging von 2011 bis 2012 eine Pilotstudie voraus, in der (1) das geplante Vorgehen auf seine praktische Umsetzbarkeit sowie (2) die im Vorfeld entwickelten Forschungsfragen auf ihre Untersuchbarkeit hin überprüft wurden (vgl. dazu Kapitel 3.1). Auf der Basis der Befunde aus der Pilotstudie wurden einige methodische Anpassungen des Forschungsdesigns vorgenommen, u.a. zur Akquirierung der Probandinnen und Probanden, zur Erhebung von (mehr und systematisierten) Hintergrundinformationen/Metadaten, zu (gestaffelten) Einverständniserklärungen und $\mathrm{zu}$ den (zielgenaueren) Geräteeinweisungen. Zudem wurden die Forschungsfragen auf empirischer Grundlage präzisiert (s.o.). Daten aus der Pilotstudie lagen auch einer ersten Veröffentlichung über Bewertungsinteraktionen in Theater-Foyergesprächen zugrunde (vgl. Hrncal \& Gerwinski 2015).

Im Interesse „einer reichhaltigen Datenbasis, die die angestrebte breite Phänomenologie der Formen der Anschlusskommunikation zu erschließen erlaubt“" (Stellungnahme des DFG-Fachkollegiums), wurden zwei unterschiedliche Theater, das Schauspiel Köln und das Apollo-Theater in Siegen, ausgewählt: Aufgrund der verschiedenen Programme beider Häuser nahmen wir an, dass sich in den Siegener und den Kölner Daten zum Teil auch unterschiedliche sprachlich-kommunikative Praktiken in den Pausengesprächen beobachten lassen, was im Interesse der Verbreiterung des Spektrums eine Erhebung an den beiden Orten sinnvoll erscheinen ließ, ohne dass die Unterschiede selbst (bisher) auf der Basis eines Vergleichs, der sich ethnomethodologisch an Beteiligtenkategorien (Ethnokategorien) festmachen müsste, zum Thema gemacht worden wären.

Um hinsichtlich der Aufführungen ein möglichst variantenreiches Spektrum abzudecken und gleichzeitig Aufführungen mit einer Pause resp. einem im Anschluss an die Aufführung stattfindenden Publikumsgespräch, bis zu dem es vorausgehend eine Pause gab, zu berücksichtigen, wurden nach Absprachen mit den Theatern jeweils verschiedenartige Produktionen ausgewählt, für die dann im Anschluss Besucherinnen und Besucher, die öfter oder regelmäßig mit anderen ins Theater gehen, gesucht und gewonnen wurden. Insgesamt wurden 43 Gespräche in 2 Theatern im Gesamtumfang von ca. 12,5 Stunden mit insgesamt 111 Probandinnen und Probanden erhoben, und zwar zu 6 Theaterinszenierungen in Köln (27 Gespräche mit ca. 8 Stunden Gesamtumfang und durch-

9 Die Kurzbeschreibung der Vorgehensweise wurde von Jan Gerwinski verfasst. Für eine ausführliche Darstellung der Datenbasis vgl. Kapitel 2, zur Methodik Kapitel 3 und 4, i.d.B. 
schnittlich etwa 18 Minuten Gesprächsdauer) und zu 7 Theaterinszenierungen in Siegen (16 Gespräche mit ca. 4,5 Stunden Gesamtumfang und durchschnittlich etwa 16,5 Minuten Gesprächsdauer). Pro Abend wurden Gespräche von maximal 3 Gruppen mit je 2 bis 4 Personen $^{10}$ aufgezeichnet. Davon wurden 37 Gespräche vollständig - mindestens als Minimaltranskripte nach GAT2 (vgl. Selting u.a. 2009) - transkribiert. 23 der Transkripte konnten als detailreichere Basistranskripte nach GAT2 aufbereitet werden.

Da sich eine Kaltakquise, das spontane Ansprechen von Besucherinnen und Besuchern unmittelbar vor der Theateraufführung, im Rahmen der Pilotstudie als schwierig und v.a. unwägbar herausgestellt hatte, wurden die persönlichen Netzwerke als Ausgangspunkt genutzt und über ein ,Schneeballsystem“ weitere Bekannte von Bekannten akquiriert. Um den Einfluss der Projektmitarbeiterinnen auf die Gespräche möglichst gering zu halten, wurde die Aufzeichnung von den Probandinnen und Probanden selbst vorgenommen. Die Probandinnen und Probanden unterschrieben eine abgestufte Einverständniserklärung, mit der sie sich entweder nur mit einer Veröffentlichung anonymisierter Gesprächsausschnitte in Schriftform oder zusätzlich mit einer namentlich anonymisierten Verwendung kurzer Audio- und Transkript-Ausschnitte in Vorträgen einverstanden erklärten. Zusätzlich konnten sich die Probandinnen und Probanden noch mit einer Veröffentlichung anonymisierter Gespräche als Audiodatum und in Schriftform (Transkripte) im Rahmen des „Forschungs- und Lehrkorpus gesprochenes Deutsch“ (FOLK) am Institut für Deutsche Sprache (IDS) in Mannheim einverstanden erklären; im Ergebnis wurden 10 Gesprächs(minimal)transkripte in FOLK übernommen. Die übrigen Daten des Projekts wurden im Rahmen eines von der Universität Siegen geförderten Projektverbundes „eHumanities“ mit Unterstützung des Zentrums für Informations- und Medientechnologie (ZIMT) nachhaltig erschlossen und langfristig archiviert.

Hinsichtlich der Datenauswertung gehen wir entsprechend der praxeologischen Orientierung unseres Projekts davon aus, dass sich im Sinne der bereits erwähnten „flachen Ontologie“ (Schatzki 2016) sprachlich-kommunikative Praktiken kleinerer und größerer Reichweite beobachten lassen, die den jeweils relevanten sozialen Kontext - verstanden als Hintergrundwissen der Beteiligten - situativ und situationsübergreifend präsupponieren (vgl. Kapitel 3.4, i.d.B., mit weiteren Referenzen). Dennoch erfolgte zusätzlich zur Gesprächsdatenerhebung eine ergänzende Ethnografie, um bei den nachfolgenden Datenaufberei-

10 Teilweise vermischten sich die Gruppen oder man traf auf weitere Bekannte, so dass es ungeplant zeitweise zu größeren Gruppen kam. Zu Gesprächsbeteiligten ohne Einverständniserklärung vgl. Kapitel 3.2. 
tungen und -auswertungen mögliche Verständnis- und Erkenntnislücken schließen zu können.

Diese Hintergrundinformationen wurden im Rahmen von Orts- und Aufführungsbesuchen, Einweisungsgesprächen und durch Auswertung der Fragenbogen gewonnen und reicherten im Rahmen der Datenaufbereitung als Metadaten die Gesprächsdaten und Transkripte an. $\mathrm{Zu}$ den erhobenen soziodemografischen Daten zählen Angaben zu Alter, Geschlecht, höchstem Bildungsabschluss, Berufsausbildung, aktuell ausgeübtem Beruf, sozialer Zugehörigkeit (wenn sich jemand explizit eine solche zuschrieb und sie damit als Teil seiner Identität präsentierte), zur Beziehung zu den anderen Gruppenmitgliedern und zur durchschnittlichen Anzahl von Theaterbesuchen pro Jahr. Hinsichtlich des allgemeinen Settings und der Theaterstücke wurden zusätzlich umfangreiche Informationen zum Spielort (Geschichte, institutionelle und räumliche Situierung, Spielstätten inkl. Innenarchitektur, Ensemble), zu ergänzenden Aufnahmebedingungen und zu den Stücken, ggf. zu Text- oder anderen Grundlagen, zu Mitwirkenden und zum Bühnenbild) sowie zu deren Aufführung (z.B. Besonderheiten der Inszenierung) protokolliert (vgl. Kapitel 2, i.d.B.).

Zur Diskussion von Zwischenergebnissen veranstaltete das Projekt in $\mathrm{Zu}$ sammenarbeit mit dem Graduiertenkolleg „Locating Media“ der Universität Siegen und dem Projekt „Handbuch Sprache in der Kunstkommunikation“ am 29.02./01.03.2016 im Apollo-Theater Siegen eine Tagung zum Thema „Alltagspraktiken des Publikums“, auf der Ergebnisse mit nationalen und internationalen Referenten im breiteren Kontext der interdisziplinären Publikumsforschung diskutiert werden konnten. ${ }^{11}$

\subsection{Forschungsgegenstände: Zum Gang der Analysen}

Im Anschluss an ausführliche Darstellungen der Datenbasis (Kapitel 2, i.d.B.) und der Methodik (Kapitel 3 und 4, i.d.B.) - einschließlich eines projektbezogenen, konstruktiven Umgangs mit dem Beobachterparadoxon - folgen empirische Untersuchungen, die jeweils bestimmten der angesprochenen Aspekte gewidmet sind. ${ }^{12}$ Zunächst stehen Praktiken des Small Talks und der (,bildungssprachlichen') Konversation im Mittelpunkt, auch in ihrem Verhältnis zur Publi-

11 Vgl. die Beiträge zur Tagung in Habscheid, Hrncal, Knipp \& Linz 2016 und Knipp, Habscheid \& Hrncal 2017.

12 Zusammenfassende Textbausteine wurden von Eva Schlinkmann (Rekonstruktive Verfahren), Christine Hrncal (Bewerten) und Jan Gerwinski (Internet) entworfen. Für ausführliche Darstellungen vgl. die entsprechenden Kapitel. 
kums- und Theaterkommunikation (Kapitel 5, i.d.B.). In diesem Zusammenhang ist u.a. die Frage zu untersuchen, welche gesprächsrhetorischen ,Skills“ - in Verbindung mit Bildungswissen - benötigt werden, um an bildungssprachlicher Konversation, hier im Theaterfoyer, sozial erfolgreich mitwirken zu können. Wie die Erkenntnisse des Projekts verdeutlichen, besteht ein zentrales rhetorisches Verfahren darin, die Themenentwicklung (vgl. dazu Linke 1988, 135f.) so zu steuern, dass sich - sei es für den Sprecher selbst, sei es für einen anderen - Möglichkeiten der Beteiligung eröffnen; man könnte hier in konversationsanalytischer Manier von ,selbst-‘ und ,fremdinitiierter' Mikro-Partizipation am Bildungsdiskurs sprechen. ${ }^{13}$ Wie bereits Dietrich Schwanitz (1999) in seiner populärwissenschaftlichen Darstellung rhetorisch zugespitzt herausarbeitete, sind in derartigen Konversationen also ,Bildung، als - stets partielles Wissen und ,Bildung als kommunikatives Können nicht scharf gegeneinander abzugrenzen, weil derjenige, der sich erfolgreich am Sprachspiel beteiligen will, durchaus über ein gewisses Bildungswissen verfügen muss (Schwanitz 1999, 399), um den mit Bildung erhobenen Anspruch, soweit dies zur Erlangung von Glaubwürdigkeit erforderlich ist, exemplarisch einlösen zu können (Schwanitz 1999, 407). Die Einsatzstellen für Bildungswissen bleiben dabei nicht dem Zufall überlassen. Vielmehr unterliegen sie der interaktionalen Themensteuerung, auf die rhetorisch geschickte Beteiligte durch Praktiken selbst- und fremdinitiierter Partizipation Einfluss zu nehmen versuchen, wobei sie gleichzeitig Anforderungen des Beziehungsmanagements bedienen. In ähnlicher Weise verfügen routinierte Beteiligte über Methoden, mit unvermeidlichen Wissenslücken rhetorisch umzugehen, „sei es durch Hinweise auf ein ähnliches Thema, durch Themenwechsel, durch interessiertes Nachfragen oder einen anspruchsvollen Scherz" (Steinig 2016, 69f.). Um mit eigenen und fremden Wissenslücken strategisch umgehen zu können, benötigt der Gebildete einen Überblick über das unhinterfragt akzeptierte (Schwanitz 1999, 397) „Bildungsterrain“ im Ganzen (Schwanitz 1999, 401), denn erst vor diesem Hintergrund können die rhetorischen Skills für Konversation erfolgreich zum Tragen kommen.

Die unterschiedlichen Verfahren, mit denen sich die Gesprächsbeteiligten auf die Aufführung und das Theater beziehen, lassen sich unter Rückgriff auf Jägers Konzept der Transkriptivität (Jäger 2002; 2008; 2012) als transkriptive Praktiken der Kunstaneignung verstehen (Kapitel 6, i.d.B.). Transkriptiv sind diese Praktiken insofern, als jeder Art der sprachlichen Aneignung eine spezifi-

13 Vgl. zur interaktionalen, multimodalen Realisierung von Partizipation unter Beteiligung eines Moderators im Kontext einer öffentlichen politischen Versammlung mit vielen Beteiligten Mondada (u.a. 2012a; 2013) und Mondada, Svensson \& van Schepen (u.a. 2015). 
sche Perspektivierung und Interpretation des Erlebten inhärent ist, die wiederum zum Anlass für interaktionale Aushandlungen der ästhetischen Erfahrungen werden kann. Schon auf der elementaren Ebene des Bezugnehmens werden nicht nur identifizierende Referenzen auf das Erlebte vollzogen (Enfield 2013), sondern durch die jeweilige Wahl der sprachlichen Mittel zugleich einzelne Aufführungsaspekte und Erfahrungen als Zuschauer relevant gesetzt und in einer je spezifischen Weise gedeutet. Verstärkt wird der transkriptive Charakter noch durch die Nachträglichkeit, mit der die kommunikative Aneignung erfolgt: Aufgrund der Depräsenz des Erlebten im Moment des Gesprächs kann ein sprachlicher Bezug auf die Aufführung, anders als etwa beim Gespräch im Museum vor dem Kunstwerk, nur im Modus der (subjektiven) Rekonstruktion (s.u.) hergestellt werden, die erst im Gespräch in intersubjektive Deutungsprozesse überführt wird.

Neben dem Moment der Nachträglichkeit der Anschlusskommunikation zeigt sich die Besonderheit des Theaters in den vielfältigen Bezugnahmen auf Aspekte der konstitutiven Performativität des Aufführungserlebnisses. Mehr und weniger ausführliche Gesprächssequenzen über das anwesende Publikum, Körperlichkeit und Stimme der Schauspieler(innen), körperliche Handlungsvollzüge auf der Bühne, Raumnutzung, Lichteffekte, eigene körperliche Empfindungen während der Aufführung und Interaktionen mit den Bühnenakteuren rücken die Aufführung als „Inbegriff des Performativen“ (Fischer-Lichte 2010, 28) in den Fokus. Zugleich lassen sie den kommunikations- und wahrnehmungsreflexiven Charakter des Theaters deutlich hervortreten. Interaktional aufgegriffen werden gerade auch solche Momente, die - seien sie inszenatorisch intendiert oder der Kontingenz des performativen Vollzugs geschuldet die Wahrnehmungsroutinen und Rahmenerwartungen als Zuschauer irritieren oder durchbrechen. Dazu gehören u.a. inszenierte mediale Wechsel (wie Tanz, Musik, Gesangseinlagen oder Videoeinspielungen), die von den reinen Sprechtheaterroutinen abweichen, aber auch nicht-intendierte Störungen wie Versprecher, falsche Sprecheinsätze o.ä., die die Aufmerksamkeit von dem aufgeführten Theaterstück und den Handlungen der Figuren auf die Darstellungs- und Schauspieltechniken lenken. Thematisiert werden zudem solche Momente, in denen sich die Beteiligten selbst als Zuschauer und Partizipierende einer Kommunikationssituation beobachten. In den Gesprächsanalysen kommt damit zum Ausdruck, wie das Theater sowohl durch ästhetisch inszenierte als auch durch der Aufführungsperformanz geschuldete Störungsmomente das Publikum dazu anregt, in der gemeinsamen Interaktion alltägliche Wahrnehmungs- und Kommunikationsgewohnheiten und mit ihnen verbundene Sinn- und Ordnungsstrukturen zu reflektieren. 
Wie bereits in Studien zur Anschluss- und Zuschauerkommunikation (vgl. u.a. Charlton \& Klemm 1998; Holly, Püschel \& Bergmann 2001; Michel 2015) sowie für den Kontext der Kunstkommunikation (vgl. u.a. Hausendorf 2005; 2007a; 2011; Hausendorf \& Müller 2016a) festgestellt wurde, wird auch in den Pausengesprächen im Theater(foyer) dem Bewerten von den Beteiligten ein hoher Stellenwert zugeschrieben (vgl. Hrncal \& Gerwinski 2015; Linz, Hrncal \& Schlinkmann 2016 und ausführlich Kapitel 7, i.d.B.): Dies ist unter anderem daran zu erkennen, dass Gesprächseinstiege in vielen Fällen über eine selbstoder fremdinitiierte Bewertung erfolgen, so dass an dieser Stelle bereits durch die Ein-Wort-Frage „und?“ fremdinitiierte Bewertungen relevant gesetzt werden.

Weiterhin zeigen die Erkenntnisse zu Bewertungsinteraktionen in unserem Datenmaterial (vgl. Kapitel 7, mit ausführlichem Literaturbericht), dass eine sequenzielle Orientierung auf - mehr oder weniger ausgeprägten - Dissens in der Pausen-Konversation zwar (phasenweise) vorkommen kann, dass (letztlich) aber zumeist die auch für Privatgespräche mehrfach beschriebene Tendenz zur Harmonisierung überwiegt (vgl. Pomerantz 1984; Auer \& Uhmann 1982; Goodwin \& Goodwin 1992). Dies schließt nicht aus, dass Chancen für und ,Rechte‘ auf die (autoritative) Beteiligung an Bewertungsinteraktionen je nach Rollenkonstellation unterschiedlich verteilt sind. Ob vor diesem Hintergrund das ,Recht auf eine (Erst-)Bewertung eher eine Chance zur Partizipation, eine mit (mehr oder weniger großen) Face-Risiken behaftete „Last“ oder beides zugleich darstellt, hängt ebenfalls von den Rollenkonstellationen sowie den je spezifischen Wissens- und Kompetenzverhältnissen ab (vgl. auch Kapitel 5). Auch die mit Bewertungen oftmals verbundene Vagheit kann vor diesem Hintergrund Beteiligungsmöglichkeiten erweitern oder einschränken.

Besonders Erstbewertungen werden oft „vorsichtiger“ realisiert, indem die Sprecher beispielsweise auf Zögerungsmarker oder Heckenausdrücke zurückgreifen, vorerst nur solche Aspekte einer Bewertung unterziehen, die kein spezielles kunsttheoretisches Wissen erfordern (u.a. das Bühnenbild, Kostüm usw.) und sich selbst sowie den anderen Beteiligten im Zuge der Bewertungsinteraktion die Art ihres Wissenszugangs sowie ihren Wissensstatus anzeigen und damit das Recht auf eine Bewertung beanspruchen, zuschreiben oder aberkennen.

Weiterhin lässt sich festhalten, dass Bewertungen in der Konversation nicht notwendigerweise begründet werden müssen und dass Begründungen vage gehalten werden können, etwa indem Referenzen nur angedeutet und nicht explizit ausgeführt werden. Eine Betrachtung der Themenverläufe zeigt, dass die an der Bewertungsinteraktion Beteiligten z.T. fließend die in den Fokus genommenen Bewertungsobjekte und -ebenen wechseln (s.o., Abschnitt 1.2) - 
„von der ästhetisch-performativen Ebene des Stücks“ „über die Handlungsebene der Figuren auf der intradiegenten Ebene mit einer moralischen Perspektivierung“ bis hin zu „eine[r] allgemeine[n] Ebene mit (moralischen) Bezügen zur Gesellschaft und zum Individuum“ (Hrncal \& Gerwinski 2015, 57).

Mit Blick auf die sequenzielle Struktur der Bewertungsinteraktionen finden sich auch andere situations- und kontextgebundene Ausprägungen, indem z.B. - dem ungezwungenen Charakter der Interaktionsorganisation im Small Talk entsprechend (vgl. Kapitel 4) - auf eine Erstbewertung gar nicht oder mit einer weiteren Erstbewertung eines anderen Sprechers reagiert wird. Nichtübereinstimmung wird von den Beteiligten auch teilweise im Sinne eines gebildeten „Besser- und Anderswissen[s]“ (Holly 2001, 433) demonstriert und bis zum Ende des Gesprächs nicht aufgehoben, dabei aber so gestaltet, dass es nicht zu gravierenden Zwischenfällen auf der Beziehungsebene kommt (s.o., Abschnitte 1.1 und 1.2). Hierbei können auch kontextuelle Rahmungen eine Rolle spielen, denn der Austausch über das im Theater Rezipierte ereignet sich zwar innerhalb der Vertrautheit der Zuschauergruppe, aber nicht etwa im heimischen Wohnzimmer, sondern an einem Ort, der auch anderen, fremden Personen prinzipiell zugänglich ist.

Bezugnahmen auf die ästhetischen Erfahrungen als Theater-Zuschauer erfolgen in Theater-Pausengesprächen wesentlich über rekonstruktive Verfahren (vgl. Kapitel 8, i.d.B.). Diese umfassen begrifflich die Gesamtheit an sprachlichkommunikativen Verfahren, die Sprecher einer Gesellschaft verwenden, um bereits vergangene Erfahrungen, Ereignisse und Handlungen in der Interaktion zu vergegenwärtigen und zu reproduzieren (vgl. Bergmann \& Luckmann 1995; Günthner 2000, 203; 2007; Gülich 2007; Gülich, Knerich \& Lindemann 2009). Rekonstruktive Verfahren reichen von freien, spontanen Gesprächsteilen, die aus wenigen Redezügen und Sequenzen bestehen, über musterhafte kleine kommunikative Formate bis hin zu umfangreichen, komplexen rekonstruktiven Gattungen, die ganze Gesprächsabschnitte dominieren. Sie repräsentieren einen Ausschnitt aus dem Gesamtkomplex des „kommunikativen Haushalts“ (Luckmann 1988), der alle kommunikativen Verfahren umfasst.

Die Bezugnahme auf ästhetische Wahrnehmungen und Erfahrungen während der Vorstellung im Theater in Form sprachlicher Rekonstruktionen kontrastiert mit dem Stellenwert der Beschreibung in der Kommunikation „,vor dem Kunstwerk“ (Hausendorf 2007). Rekonstruktionen in Pausengesprächen lassen sich u.a. in Bezug auf ihren Gegenstand (Theaterereignis - mit weiteren Unteraspekten; anderes Medienereignis; Welt außerhalb des Theaters), den relativen Wissensstatus der Beteiligten (informierend vs. kommemorierend) und den 
Darstellungsmodus (berichtend vs. re-inszenierend) untergliedern (vgl. Kapitel 8, i.d.B.; Ulmer \& Bergmann 1993).

Wie die Analysen der Gesprächsdaten zeigen, werden Rekonstruktionen typischerweise sukzessiv in der Gruppe ko-produziert und teilweise ,ausgehandelt', als transkriptive Verfahren gehen sie stets mit einer Deutung bzw. Umdeutung der Inhalte einher, auf die sie sich beziehen (vgl. Kapitel 6, i.d.B.). Die sprachlich-kommunikativen Einstiege in die Rekonstruktionen eröffnen den jeweiligen Handlungszusammenhang, in den die Rekonstruktionen eingebettet sind, neben dem deutenden z.B. einen bewertenden, erläuternden oder argumentativen (vgl. Hausendorf \& Müller 2016a, 5f.), aber auch einen gemeinschaftsstiftenden, unterhaltenden oder gesprächserhaltenden Kontext. Gesellige Kommunikation auf der einen und Kunstkommunikation auf der anderen Seite schließen sich also nicht notwendig aus, sondern können sich auch überschneiden, indem etwa gesellige Kommunikation den gesprächsorganisatorischen und beziehungsbezogenen Rahmen dafür bietet, dass die Verhandlung von Kunst in einem kooperativen Kontext überhaupt stattfinden kann (vgl. Kapitel 5).

Mehrdeutige Bezugnahmen und Rekonstruktionen einzelner Aspekte des Zuschauererlebnisses können einen Anlass bieten, von der Ebene der ästhetischen Erfahrung und des Dargestellten auf die Ebene der gesellschaftlichen Wirklichkeit zu wechseln - die Theatererfahrung ins reale Leben $\mathrm{zu}$ „übertragen“ - und das Gespräch auf gesellschaftspolitisch bzw. persönlich relevante Themen zu lenken (Linz 2016): Vielfach werden zunächst vage Referenzialisierungen in - auch kooperativ vollzogenen - Elaborationen erst zunehmend spezifiziert oder modifiziert. Insbesondere pronominale Referenzen können aufgrund ihrer Uneindeutigkeit dabei auch als Gelenkstelle fungieren, an der ohne sprachliche Explizierung ein Perspektivwechsel vollzogen wird, etwa von den Darstellern zu den Figuren und vice versa oder von der Aufführung zur Alltagswelt (vgl. Hrncal \& Gerwinski 2015).

Eine Perspektive für Folgeuntersuchungen wird in einem Ausblick (Kapitel 9, i.d.B.) aufgezeigt: So lässt sich, etwa im Blick auf Strukturen von (Halb-) Öffentlichkeit, neben der Foyer- und Pausenkommunikation unter räumlich Anwesenden auch die - parallele und/oder nachfolgende - Anschlusskommunikation in sozialen Medien und anderen Kommunikationsformen untersuchen und zu den Foyer-/Pausengesprächen in Beziehung setzen. 